\title{
Antimicrobial and Antivirulence Action of Eugenia brejoensis Essential Oil in vitro and in vivo Invertebrate Models
}

\section{OPEN ACCESS}

Edited by:

Kin Weng Kong,

University of Malaya, Malaysia

Reviewed by:

Jianhua Wang,

Feed Research Institute (CAAS),

China

Nayeli Alva-Murillo,

University of Guanajuato, Mexico

${ }^{*}$ Correspondence:

Clovis Macêdo Bezerra Filho

clovisfilho@gmail.com

Luís Cláudio Nascimento da Silva

luisclaudionsilva@yahoo.com.br

Maria Luiza Vilela Oliva

mlvoliva@unifesp.br

Specialty section:

This article was submitted to

Antimicrobials, Resistance

and Chemotherapy,

a section of the journal

Frontiers in Microbiology

Received: 19 October 2019

Accepted: 27 February 2020

Published: 19 March 2020

Citation:

Bezerra Filho CM, da Silva LCN,

da Silva MV, Løbner-Olesen A, Struve C, Krogfelt KA, Correia MTS

and Vilela Oliva ML (2020)

Antimicrobial and Antivirulence Action

of Eugenia brejoensis Essential Oil

in vitro and in vivo Invertebrate

Models. Front. Microbiol. 11:424.

doi: 10.3389/fmicb.2020.00424

\section{Clovis Macêdo Bezerra Filho ${ }^{1,2 *}$, Luís Cláudio Nascimento da Silva ${ }^{3 *}$, Márcia Vanusa da Silva ${ }^{1}$, Anders Løbner-Olesen ${ }^{4}$, Carsten Struve ${ }^{5}$, Karen Angeliki Krogfelt ${ }^{5,6}$, Maria Tereza dos Santos Correia ${ }^{1}$ and Maria Luiza Vilela Oliva ${ }^{2 *}$}

'Biochemistry Department, Federal University of Pernambuco, Recife, Brazil, ${ }^{2}$ Biochemistry Department, Federal University of São Paulo, São Paulo, Brazil, ${ }^{3}$ Programa de Pós-graduação em Biologia Microbiana, CEUMA University, São Luís, Brazil, ${ }^{4}$ Department of Biology, University of Copenhagen, Copenhagen, Denmark, ${ }^{5}$ Department of Bacteria, Parasites and Fungi, Staten Serum Institut, Copenhagen, Denmark, ${ }^{6}$ Department of Science and Environment, Roskilde University, Roskilde, Denmark

Eugenia brejoensis L. (Myrtaceae) is an endemic plant from caatinga ecosystem (brazilian semi-arid) which have an E. brejoensis essential oil (EbEO) with reported antimicrobial activity. In this work, in vitro and in vivo models were used to characterize the inhibitory effects of EbEO in relation to Staphylococcus aureus. EbEO inhibited the growth of all tested $S$. aureus strains (including multidrug resistance isolates) with values ranging from 8 to $516 \mu \mathrm{g} / \mathrm{mL}$. EbEO also synergistically increased the action of ampicillim, chloramphenicol, and kanamycin. The treatment with subinhibitory concentrations (Sub-MIC) of EbEO decreased S. aureus hemolytic activity and its ability to survive in human blood. EbEO strongly reduced the levels of staphyloxanthin (STX), an effect related to increased susceptibility of $S$. aureus to hydrogen peroxide. The efficacy of EbEO against $S$. aureus was further demonstrated using Caenorhabditis elegans and Galleria mellonella. EbEO increased the lifespan of both organisms infected by $S$. aureus, reducing the bacterial load. In addition, EbEO reduced the severity of S. aureus infection in G. mellonella, as shown by lower levels of melanin production in those larvae. In summary, our data suggest that EbEO is a potential source of lead molecules for development of new therapeutic alternatives against $S$. aureus.

Keywords: Caenorhabditis elegans, Galleria melonella, infections models, multidrug resistance, natural products, Staphylococcus aureus, virulence factors

\section{INTRODUCTION}

Staphylococcus aureus is the etiologic agent of a wide spectrum of clinical conditions ranging from superficial skin infections and soft tissue infections to severe sepsis (Asgeirsson et al., 2018; Mcneil and Fritz, 2019; Turner et al., 2019). The indiscriminate use of antibiotic has induced the emergence of multidrug resistant (MDR) strains that have spread from hospital settings to different environmental and victimized various individuals (Mcneil and Fritz, 2019; Turner et al., 2019). 
Indeed, both hospital- and community-associated S. aureus strains display complex combinations of virulence and resistance genes and have been related to increased ratios of morbidly and mortality and represent serious concerns for global systems worldwide (De Carvalho et al., 2019; Liang et al., 2019; Vestergaard et al., 2019). The major drugs currently used against methicillin-resistant $S$. aureus (MRSA) are vancomycin, teicoplanin, linezolid, and daptomycin (Werth et al., 2014); however, isolates with resistance or low susceptibility have been detected for all these drugs (Endimiani et al., 2011; Kos et al., 2012; Capone et al., 2016; Bakthavatchalam et al., 2017). Taken together, these data justify the search for new alternatives for the treatment of infections caused by $S$. aureus, and plant-derived products are highlighted as interesting candidates (Dos Santos et al., 2016; Mittal et al., 2018).

Plants are known to produce a large variety of molecules with interest for pharmaceutic and food industries, among them are the volatile compounds present in the essential oils (EOs) (Alves et al., 2017; Mittal et al., 2018; Stevanovic et al., 2018; Taghavi et al., 2018; Rao et al., 2019). EOs are complex mixture of secondary metabolites produced by aromatic plants usually involved in their protection against pathogens (Mittal et al., 2018). Several EOs have been demonstrated as efficient antimicrobial agents able to inhibit different MDR strains and to improve the activity of antibiotics (Langeveld et al., 2014; Mittal et al., 2018; Rao et al., 2019). The EOs components can interact with multiple targets in bacteria such as membrane, proteins synthesis, as well as inhibit efflux pumps and virulence related pathways (such as biofilm formation and toxin production) (Dos Santos et al., 2016; Kim et al., 2016; Kong et al., 2016b; Kang et al., 2018; Mittal et al., 2018; Rubini et al., 2018; Espinoza et al., 2019).

Eugenia brejoensis is an EO-bearing plant from Mytaceae family which has been described as an endemic species in Brazil (present in the states of Pernambuco, Sergipe, Alagoas, Paraíba, Espirito Santo) (Mazine and Souza, 2008; Giaretta and Peixoto, 2014; Mendes et al., 2018). The E. brejoensis EO (EbEO) is mainly composed by sesquiterpenes (such as $\delta$-cadinene, $\beta$-caryophyllene, and $\alpha$-muurolol) (Da Silva et al., 2015). EbEO has shown inhibitory actions against Aedes aegypti larvae (Da Silva et al., 2015) and Trypanosoma cruzi (Oliveira De Souza et al., 2017). In relation to antibacterial activity, nanoemulsions of EbEO were able to inhibit the growth of Pseudomonas fluorescens (Mendes et al., 2018). The present work aims to demonstrate the efficacy of EbEO against $S$. aureus using in vitro and in vivo invertebrate models (Caenorhabditis elegans and Galleria mellonella).

\section{MATERIALS AND METHODS}

\section{Plant Material}

Leaves of E. brejoensis were collected at Parque Nacional do Catimbau (Pernambuco, Brazil) on dry season (September, 2015). All the plant material was processed following the usual techniques in taxonomy and deposited in the Herbarium of Instituto Agronomico de Pernambuco (voucher access number: IPA 84.033). The EO was obtained from leaves of E. brejoensis
(EbEO) by hydrodistillation as previously reported (Da Silva et al., 2015). The oil used in this study was characterized by gas chromatography-mass spectrometry (GC/MS) and the chemical profile was published by Da Silva et al. (2015).

\section{Bacterial Strains Used in the Study}

The standard strain S. aureus ATCC 29312 was used in most of the assays. The antimicrobial activity of EbEO was further analyzed against a collection of clinical isolates of S. aureus deposited in the Microbial Collection of Departamento de Antibioticos from Universidade Federal de Pernambuco (UFPEDA). The antibiotic resistance profile of each strain is shown in Table 1. The strains used in the present study are part of Dr. Anders Løbner-Olesen collection at University of Copenhagen. The expression of virulence- and SOS-related genes ( $h l a, s p a$, or $r e c A$ ) was performed using strains carrying the targeted genes fused with lacZ (which encodes for $\beta$-galactosidase) (Nielsen et al., 2010; Gottschalk et al., 2013). The strains in Table 1 were kindly shared by Prof. Hanne Ingmer, Copenhagen University.

\section{Determination of Minimum Inhibitory Concentration}

The antimicrobial activity of EbEO was determined against the standard strains S. aureus ATCC 29312 and clinical isolates (Table 1). Serial dilutions (1024 to $2 \mu \mathrm{g} / \mathrm{mL}$ ) of EbEO were prepared in 96-wells plates containing Luria-Bertani (LB) broth. Each well received $10 \mu \mathrm{L}$ of a microbial suspension [bacterial load of approximately $1.0 \times 10^{7}$ colony forming units per milliliter $(\mathrm{CFU} / \mathrm{mL})$ for each well]. The plates were incubated at $37^{\circ} \mathrm{C}$, and after $24 \mathrm{~h}$ each well received $30 \mu \mathrm{L}$ of $0.03 \%$ resazurin sodium solution (Sigma-Aldrich ${ }^{\circledR}$ ). Following, the plates were incubated for $40 \mathrm{~min}$ and the minimum inhibitory concentration (MIC) was defined as the lowest concentration capable of inhibiting bacterial growth (as evaluated color change).

\section{Time-Kill Studies}

Overnight cultures of $S$. aureus ATCC 29312 were diluted $1: 100$ in $\mathrm{LB}$ broth and placed in a shaking water bath at $37^{\circ} \mathrm{C}$ until an optical density at $600 \mathrm{~nm}\left(\mathrm{OD}_{600}\right)$ of 0.1 was reached. This microbial suspension was distributed in fresh LB broth containing EbEO (128 or $1024 \mu \mathrm{g} / \mathrm{mL}$; corresponding to $2 \times$ MIC or $8 \times$ MIC, higher concentrations was applied to verify action of concentrations greater than MIC50). Bacteria treated with ciprofloxacin $(2 \mu \mathrm{g} / \mathrm{mL} ; 2 \times \mathrm{MIC})$ or without treatment were used as positive and negative controls, respectively. Cell growth was monitored in specific time points $(0,1,2,3,4$, and $5 \mathrm{~h}$ ) by plating $4 \mu \mathrm{L}$ of 10 -fold-diluted suspensions from each tube in quadruplicate. The plates were incubated at $37^{\circ} \mathrm{C}$ for $24 \mathrm{~h}$. After this period, the colonies were counted for the calculation of $\mathrm{CFU} / \mathrm{mL}$.

\section{Combinatory Effects}

The interaction between EbEO and some important clinical used drugs (ampicillin-25 $\mu \mathrm{g} / \mathrm{mL}$, ciprofloxacin $-0.78 \mu \mathrm{g} / \mathrm{mL}$, chloramphenicol-12.5 $\mu \mathrm{g} / \mathrm{mL}$, erythromycin $-0.39 \mu \mathrm{g} / \mathrm{mL}$, and 
TABLE 1 | Antimicrobial effects of EbEO in association to antibiotics.

\begin{tabular}{|c|c|c|c|}
\hline S. aureus & Source & Resistance profile & MIC $(\mu \mathrm{g} / \mathrm{mL})$ \\
\hline ATCC 29213 & Standard strain & - & 128 \\
\hline UFPEDA 02 (= ATCC 6538) & Standard strain & - & 256 \\
\hline UFPEDA 659 & Catheter tip & NAL/OXA & 128 \\
\hline UFPEDA 671 & Bone fragment & AMI/AMP/CIP/CLI/CLO/GEN/NAL/OXA/TET/TRI & 512 \\
\hline UFPEDA 679 & Surgical wound secretion & AMI/CLI/AMP/CFL/CFZ/NAL/OXANAN & 128 \\
\hline UFPEDA 683 & Purulent exudate & AMI/AMP/CIP/CFL/CFO/CFZ/CLI/CLO/CPM/CRX/CTX/GEN/NAL/OXA/TRI/NAN & 8 \\
\hline UFPEDA 691 & Catheter tip & $\mathrm{CIP} / \mathrm{CLO} / \mathrm{NAL}$ & 128 \\
\hline UFPEDA 705 & Surgical wound & AMP/CFL/CFO/CPM/CRX/NAL/NIT/OXA/GEN & 256 \\
\hline UFPEDA 726 & Nasal secretion & AMP/CIP/CLO/GEN/OXA/TRI & 128 \\
\hline UFPEDA 731 & Surgical wound secretion & AMP/CFL/CFO/CLI/CLO/CRX/CIP/GEN/NAL/OXA/TRI & 512 \\
\hline UFPEDA 802 & Nasal secretion & AMI/AMP/OXA/CFL/CFO/CFZ/CIP/CLI/CLO/CPM/CRX/CTX/GEN/NAL/TET/TRI & 512 \\
\hline
\end{tabular}

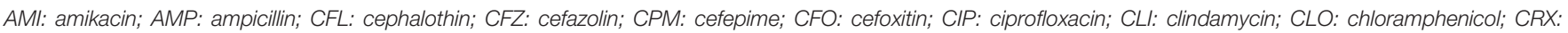

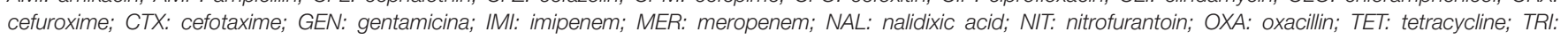
trimethoprim; VAN: vancomycin. MICs were obtained using the microdilution method as described.

kanamycin-6.25 $\mu \mathrm{g} / \mathrm{mL}$ ) were evaluated using checkerboard assay against $S$. aureus ATCC 29312. Fractional inhibitory concentration index (FICI) was assessed algebraically using the following equation:

$$
\mathrm{FICI}=\mathrm{FIC}_{\mathrm{EbEO}}+\mathrm{FIC}_{\mathrm{D}}=\left(\mathrm{EbEO} / \mathrm{MIC}_{\mathrm{EbEO}}\right)+\left(\mathrm{D} / \mathrm{MIC}_{\mathrm{D}}\right)
$$

Where "EbEO" is the concentration $(\mu \mathrm{g} / \mathrm{mL})$ of EbEO in a given well, and $\mathrm{MIC}_{\mathrm{EbEO}}$ represents the control MIC of EbEO alone. " $\mathrm{D}$ " is the concentration of the tested drug in a given well, and $\mathrm{MIC}_{\mathrm{D}}$ represents the MIC of the tested drug alone. The interactions were defined as: (i) synergistic if FICI $\leq 0.5$; (ii) additive if $0.5<$ FICI $\leq 1$; (iii) non-interaction if $1<$ FICI $<4$; and (iv) antagonistic if FICI $\geq 4$ (Da Silva et al., 2016).

\section{Expression of Virulence- and SOS-Related Genes Through $\beta$-Galactosidase Activity}

The effects of EbEO on the expression of genes related to virulence and SOS pathways were performed using $S$. aureus 8325-4 derivative strains carrying the targeted genes ( $h l a, s p a$, or $\operatorname{rec} A$ ) fused with $\operatorname{lac} Z$ (which encodes for $\beta$-galactosidase) (Vestergaard et al., 2015). In all assays, each strain was exponentially grown in $\mathrm{LB}$ medium until an $\mathrm{OD}_{600}$ between 0.1 and 0.2. Cells were treated with EbEO $(64 \mu \mathrm{g} / \mathrm{mL}$, $0.5 \times \mathrm{MIC})$, and permeabilizated by toluene $(1 \mathrm{~mL})$ after $3 \mathrm{~h}$. The $\beta$-galactosidase activity was measured using ONPG (ortho-nitrophenyl- $\beta$-galactoside; Sigma-Aldrich). In the assays for $\operatorname{rec} A$ expression, ciprofloxacin $(0.5 \mu \mathrm{g} / \mathrm{mL})$ was used as positive control.

\section{Evaluation of Staphyloxanthin (STX) Inhibition}

Overnight cultures of $S$. aureus ATCC 29312 were diluted (1:100) in LB medium and samples $(1 \mathrm{~mL})$ of this suspension were incubated with sub-inhibitory concentrations of $\operatorname{EbEO}(64,32$, and $16 \mu \mathrm{g} / \mathrm{mL}$, corresponding, respectively, to $0.125 \times \mathrm{MIC}$, $0.25 \times \mathrm{MIC}$, and $0.5 \times \mathrm{MIC})$. After overnight incubation at $37^{\circ} \mathrm{C}$, the tubes were centrifuged $(10,000 \mathrm{r} / \mathrm{min}$ for $10 \mathrm{~min})$, suspended with $1 \mathrm{~mL}$ of phosphate-buffered saline (PBS), and recentrifuged. Bacteria pellets were then photographed. Following the total carotenoid pigments [including staphyloxanthin (STX)] in each pellet were extracted using in methanol $(0.2 \mathrm{~mL})$ and incubated for $3 \mathrm{~min}$ at $55^{\circ} \mathrm{C}$. The methanol phase (supernatant) and cell debris were separated by centrifugation $(10,000 \mathrm{r} / \mathrm{min}$ for $10 \mathrm{~min}$ ) and the pellets were submitted to entire pigment extraction procedure three more times. Finally, the absorbance was determined at $465 \mathrm{~nm}$ (Silva et al., 2017).

\section{Effect of EbEO on Susceptibility of S. aureus to Hydrogen Peroxide}

Microbial suspension (standardized at $\mathrm{OD}_{600}=1.0$; Liu et al., 2005) prepared from overnight cultures of S. aureus ATCC 29312 were incubated with sub-inhibitory concentrations of EbEO (64, 32 , and $16 \mu \mathrm{g} / \mathrm{mL}$, corresponding, respectively, to $0.125 \times \mathrm{MIC}$, $0.25 \times \mathrm{MIC}$, and $0.5 \times \mathrm{MIC})$. Each culture received $\mathrm{H}_{2} \mathrm{O}_{2}$ to reach a final concentration of $1.5 \%(\mathrm{v} / \mathrm{v})$ for $60 \mathrm{~min}$ and were incubated at $37^{\circ} \mathrm{C}$. The percentage of cells surviving the stresses was calculated as $\mathrm{CFU} / \mathrm{mL}$ remaining after each stress divided by the initial $\mathrm{CFU} / \mathrm{mL}$.

\section{Anti-hemolytic Evaluation}

Staphylococcus aureus ATCC 29312 overnight cultures were diluted at 1:100 in fresh LB and cultured with or without subinhibitory concentrations of EbEO (64 and $32 \mu \mathrm{g} / \mathrm{mL}$ ) at $37^{\circ} \mathrm{C}$. After $16 \mathrm{~h}$ (to guarantee a constant number of bacteria present in experiment), $50 \mu \mathrm{L}$ of culture supernatant was added to $1 \mathrm{~mL}$ of $3 \%$ human erythrocytes solution. The mixture was incubated at $37^{\circ} \mathrm{C}$ for $1 \mathrm{~h}$ with $250 \mathrm{r} / \mathrm{min}$ shaking. The supernatant was collected by centrifugation at $10,000 \mathrm{r} / \mathrm{min}$ for $10 \mathrm{~min}$, and the optical density was measured at $540 \mathrm{~nm}$.

\section{Bacterial Activity in Whole Blood}

To verify whether EbEO could enhance the antimicrobial action in whole blood, the S. aureus ATCC 29312 (diluted in LB medium; 1:100) was grown in the presence of EbEO (64 and $32 \mu \mathrm{g} / \mathrm{mL}$ ) at $37^{\circ} \mathrm{C}$ with shaking of $250 \mathrm{r} / \mathrm{min}$. After $6 \mathrm{~h}$, the $S$. 
aureus suspension $(62.5 \mu \mathrm{L})$ with $\mathrm{EbEO}(64$ or $32 \mu \mathrm{g} / \mathrm{mL})$ were mixed with freshly drawn human whole blood $(0.5 \mathrm{~mL})$ and reincubated in the conditions described above for $2 \mathrm{~h}$. The samples were plated in LB agar and the survival was measured by counting viable colonies (expressed as CFU/mL) (Lee et al., 2013).

\section{Infectious Model With Caenorhabditis elegans}

The infection model was performance using C. elegans AU37, a temperature-sensitive sterile strain [sek-1(km4); glp-4(bn2) I; MAPK kinase deficiency] (Jakobsen et al., 2013). The strain was propagated on nematode growth medium (NGM) containing Escherichia coli OP50 as food source. Prior each assay, the worms were age-synchronized by bleaching with alkaline hypochlorite and sodium hydroxide. The released embryos were placed on NGM plates at $25^{\circ} \mathrm{C}$ (this temperature does not allow the reproduction) until reached the young adult stage. At this time, 15 larvae were transferred to 24-wells plates containing the M9 liquid medium and overnight culture S. aureus ATCC 29312 (grown in LB medium containing $10 \mu \mathrm{g} / \mathrm{mL}$ cholesterol) in a ratio of $4: 1$ (v/v) (Kong et al., 2014). EbEO (128, 64, and $32 \mu \mathrm{g} / \mathrm{mL}$ ) was added and the worm longevity was assessed every day. OP 50 was used with positive control (worm and E. coli) and $S$. aureus was used with negative control (worm and ATCC 29312). The animals were classified as dead when they did not present spontaneous movement or response after stimulation with a platinum loop. All experiments were performed according to Wormbook (Stiernagle, 2006).

\section{Infectious Model With Galleria mellonella}

Galleria mellonella larvae ( $200 \mathrm{mg}$ ) were randomly distributed in three experimental groups $(n=10)$ with or without oil treatment. Two groups were infected by injection of $10 \mu \mathrm{L}$ of a recent $S$. aureus ATCC 29312 suspension $\left(1.0 \times 10^{5} \mathrm{CFU}\right.$; different bacterial concentrations was previously optimized to guarantee an inicial sublethal load to this assay), into the last left proleg, followed by incubation at $37^{\circ} \mathrm{C}$. After $2 \mathrm{~h}$, one of these groups received $10 \mu \mathrm{L}$ of $128 \mu \mathrm{g} / \mathrm{mL}$ EbEO (resulting in dose of $6.4 \mathrm{mg} / \mathrm{kg}$ ). Larvae treated with PBS (vehicle) were used as positive control.

\section{Quantification of S. aureus Load in G. mellonella Hemolymph}

Galleria mellonella larvae were infected with $S$. aureus ATCC 29312 and treated with EbEO as described above. Each day, a total of five larvae were cut in the cephalocaudal direction with a scalpel blade and squeezed to remove the hemolymph. Each sample was 10-fold-diluted in PBS and $4 \mu \mathrm{L}$ was plated on LB agar. After $24 \mathrm{~h}$-incubation at $37^{\circ} \mathrm{C}$, the colonies were enumerated, and the results expressed as $\mathrm{CFU} / \mathrm{mL}$.

\section{Quantification of Hemolymph Melanization}

Melanization test concerns the production of this pigment under stress conditions. G. mellonella $(n=10)$ were infected with S. aureus ATCC $29312\left(10^{6}\right.$ cells/larva) aimed to evaluate melanization as a response to higher concentrations of bacteria (stress) and to quantify this response as a function of the melanin/time correlation after being treated with $6.4 \mathrm{mg} / \mathrm{kg}$ EbEO. After the incubation ( 1 or $3 \mathrm{~h}$ ), the hemolymph was collected and diluted $(1: 10 ; \mathrm{v} / \mathrm{v})$ in cold PBS. The cells suspensions were centrifuged at $12,000 \mathrm{r} / \mathrm{min}$ and the absorbance of each supernatant was determined at $465 \mathrm{~nm}$.

\section{Statistical Analysis}

All assays were performed in triplicate in at least two independent experiments. Statistical analyses were performed using the

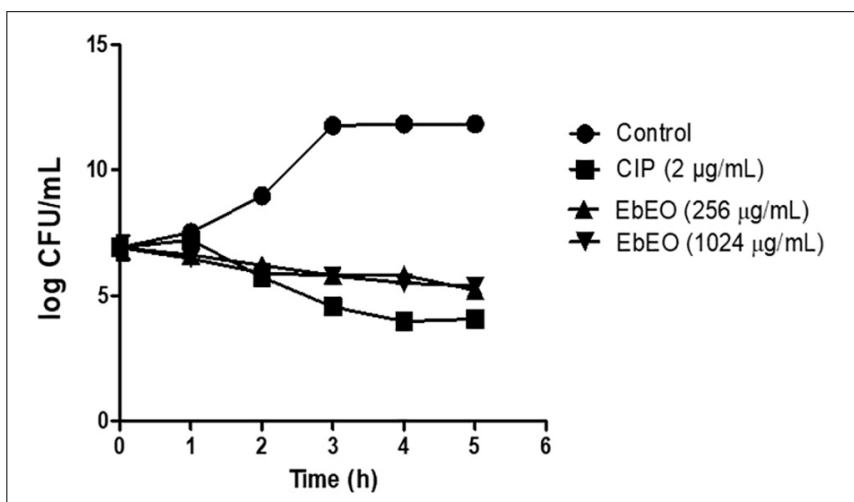

FIGURE 1 | Effects of Eugenia brejoensis essential oil (EbEO) on growth of Staphylococcus aureus. S. aureus ATCC 29312 was treated with $2 \times$ MIC $(256 \mu \mathrm{g} / \mathrm{mL})$ or $8 \times$ MIC EbEO $(1024 \mu \mathrm{g} / \mathrm{mL})$. Bacteria treated with ciprofloxacin (CIP at $2 \mu \mathrm{g} / \mathrm{mL} ; 2 \times$ MIC) or without treatment were used as positive and negative controls, respectively. Samples were taken every hour to assess bacterial viability.

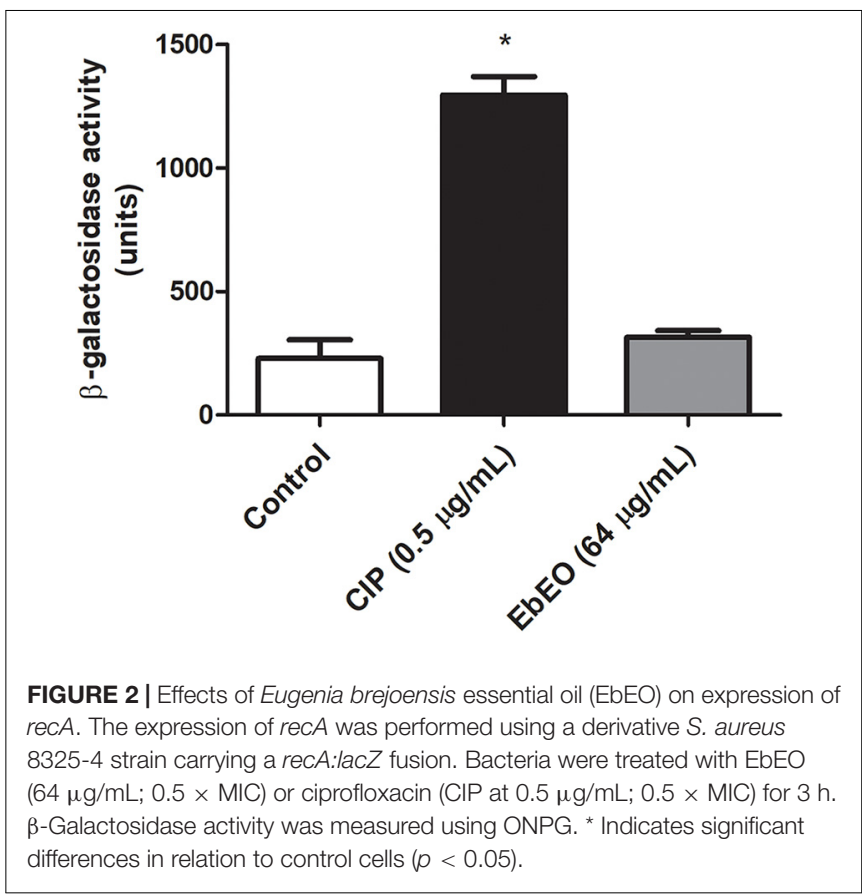


software GraphPad Prism version 7.' Data were analyzed by one-way, two-way analysis of variance (ANOVA), and Tukey test. A $p$-value of $<0.05$ was considered as statistically significant. Differences in the survival were determined using the Kaplan-Meier method and log-rank test was used to compare survival curves.

\section{RESULTS}

\section{EbEO Enhances the Activity of Drugs Toward S. aureus}

Initially, we performed a microdilution-based assay to evaluate antimicrobial activity of EbEO against all tested S. aureus strains, including those with multidrug resistance profile (MDR strains). This oil inhibited the standard S. aureus ATCC 29312 with an MIC of $128 \mu \mathrm{g} / \mathrm{mL}$, while the MIC values for the other strains

${ }^{1}$ www.graphpad.com ranged from 8 to $516 \mu \mathrm{g} / \mathrm{mL}$. The $\mathrm{MIC}_{50}$ (concentration able to inhibit $50 \%$ of the tested strains) was $128 \mu \mathrm{g} / \mathrm{mL}$ (Table 1).

Following, a time-kill study was performed using EbEO at $128 \mu \mathrm{g} / \mathrm{mL}(2 \times \mathrm{MIC})$ or $1024 \mu \mathrm{g} / \mathrm{mL}(8 \times \mathrm{MIC})$. Both oil concentrations were able to inhibit the growth of S. aureus ATCC 29312 without any significant differences among them. After $3 \mathrm{~h}$, the oil induced reductions of $50 \%$ in the number of viable colonies when compared to untreated cells. The oil exhibited a profile, similar to the observed from ciprofloxacin $(0.5 \mu \mathrm{g} / \mathrm{mL})$ (Figure 1). Further, the action of EbEO was not associated with an increased expression of $\operatorname{rec} A$, the first gene related with the activation of SOS response, a pathway involved in the DNA repair (Vestergaard et al., 2015) (Figure 2).

Next, we determined whether EbEO could improve the action of some antibiotics (from different classes) toward $S$. aureus. EBEO synergistically increased the action of ampicillin ( $\Sigma$ FIC: 0.45 ), chloramphenicol ( $\Sigma$ FIC: 0.15 ), and kanamycin ( $\Sigma$ FIC: 0.075 ), while it had additive effects with ciprofloxacin ( $\Sigma$ FIC: 0.6$)$ and erythromycin ( $\Sigma$ FIC: 0.6 ).
A
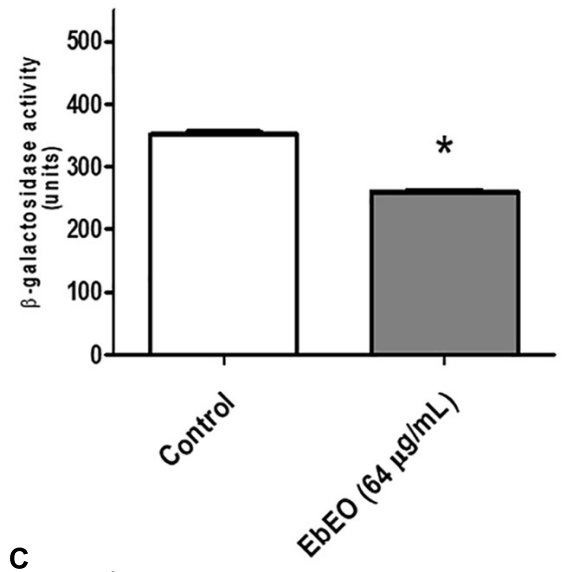

C

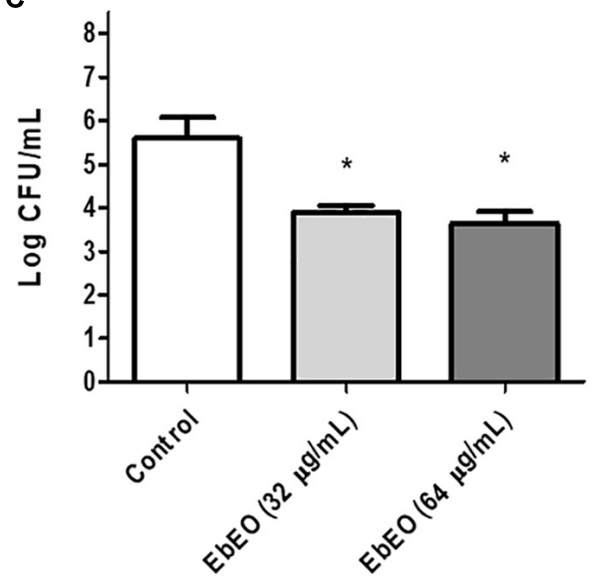

B
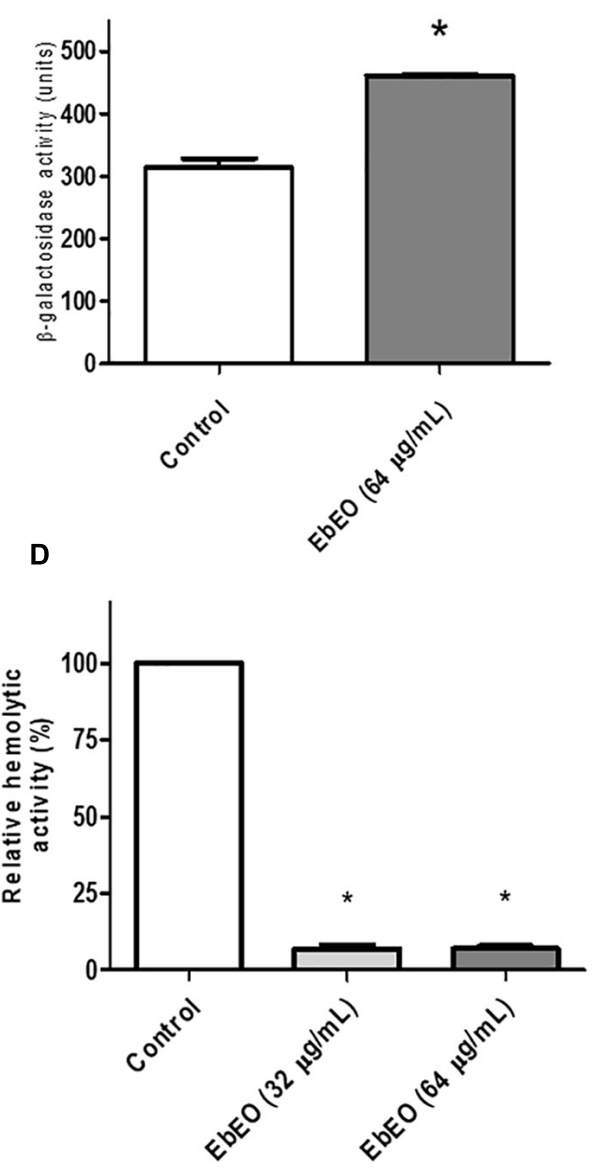

FIGURE 3 | Influence of Eugenia brejoensis essential oil (EbEO) on some virulence properties of S. aureus. (A) Effects of EbEO on expression of hla using S. aureus 8325-4 derivative strains carrying hla:lacZ. (B) Effects of EbEO on expression of spa using S. aureus 8325-4 derivative strains carrying spa:lacZ. (C) Interference of EbEO on hemolytic activity of $S$. aureus. (D) Inhibition of EbEO on S. aureus survival on whole blood. * Indicates significant differences in relation to control cells $(p<0.05)$. 


\section{EbEO Affects the Expression of Quorum Sensing-Related Genes of S. aureus and Its Hemolytic Activity}

Following, we evaluated the effects of EbEO on the expression of two gene of this system ( $h l a$ and spa that encode alpha-hemolysin and protein A, respectively). It is expected that a quorum sensing inhibitor (QSI) reduces the expression of hla and increases the transcriptional levels of spa (Nielsen et al., 2010). Based on this, the results indicated the EbEO (at $0.5 \times$ MIC) could alter the expression of hla (downregulation) and spa (upregulation), suggesting that this oil is QSI (Figures 3A,B).

We further analyzed whether EbEO could decrease the hemolytic activity of $S$. aureus and its ability to survive in human blood. The treatment with $\mathrm{EbEO}(0.5 \times \mathrm{MIC}$ or $0.25 \times \mathrm{MIC})$ strongly inhibited the hemolysis-mediated by $S$. aureus (around 90\% of inhibition) (Figure 3C). Similarly, the survival of this pathogen on blood was impaired by subinhibitory concentrations (Sub-MIC) values of EbEO (Figure 3D).

\section{EbEO Inhibits the Production of Staphyloxanthin and Increases the Susceptibility of $S$. aureus to Hydrogen Peroxide}

Figure 4A shows that EbEO strongly reduced the levels of staphyloxanthin in a dose-dependent manner $(95.63 \pm 0.98$ and $89.38 \pm 1.49 \%$ for $\mathrm{EbEO}$ at 64 or $32 \mu \mathrm{g} / \mathrm{mL}$, respectively). In addition, the treatment with $\mathrm{EbEO}$ (at 64 or $32 \mu \mathrm{g} / \mathrm{mL}$; $0.5 \times \mathrm{MIC}$ or $0.25 \times \mathrm{MIC})$ potentialized the toxicity of hydrogen peroxide $(1.5 \% \mathrm{v} / \mathrm{v})$ toward $S$. aureus (Figure $4 \mathrm{~B}$ ). Similar levels of viability reduction were observed for both concentrations $(24.12 \pm 4.19$ and $20.87 \pm 0.87 \%$ for $\mathrm{EbEO}$ at 64 and $32 \mu \mathrm{g} / \mathrm{mL}$, respectively), when compared to cells treated with hydrogen peroxide alone $(p<0.05)$. These results suggest that the inhibition of staphyloxanthin production by EbEO impaired the antioxidant system of $S$. aureus and increased its susceptibility to oxidant attack. Based on these findings, we decided to evaluate the anti-infective efficacy of EbEO using two alternative experimental models: C. elegans and G. mellonella.

\section{EbEO Increases the Lifespan of C. elegans Infected by S. aureus}

The infection of $C$. elegans by $S$. aureus resulted in a reduction of worm viability and 8 days post-infection (dpi) the survival ratio of this group was 30\%, while the uninfected animals showed $90 \%$ survival at this day (Figure 5A). The median survival of S. aureus-infected worms was 7 days. This scenario was radically changed by the treatment with EbEO at 128 or $64 \mu \mathrm{g} / \mathrm{mL}$. The animals treated with $128 \mu \mathrm{g} / \mathrm{mL}$ EbEO showed survival curve similar to control group ( $p>0.05)$. In the end ( $8 \mathrm{dpi})$, the survival of worms treated with EbEO were around 60 and $80 \%$, for concentrations of 64 or $128 \mu \mathrm{g} / \mathrm{mL}$, respectively (Figure 5A). In addition, the $C$. elegans larvae treated with $128 \mu \mathrm{g} / \mathrm{mL}$ EbEO (MIC) also exhibited lower bacterial load than those incubated with $64 \mu \mathrm{g} / \mathrm{mL} \mathrm{EbEO} \mathrm{(Figure} \mathrm{5B).} \mathrm{It} \mathrm{is} \mathrm{important} \mathrm{to} \mathrm{highlight}$ that the tested EbEO concentrations did not significantly reduce

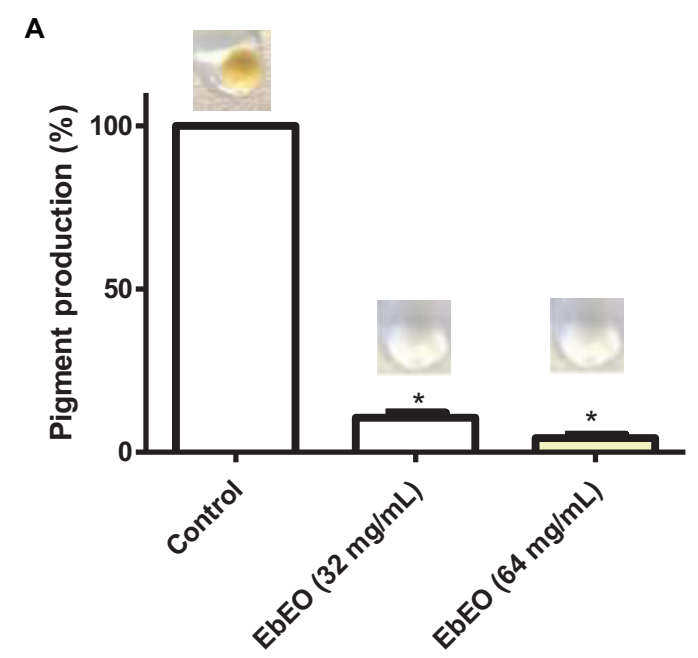

B

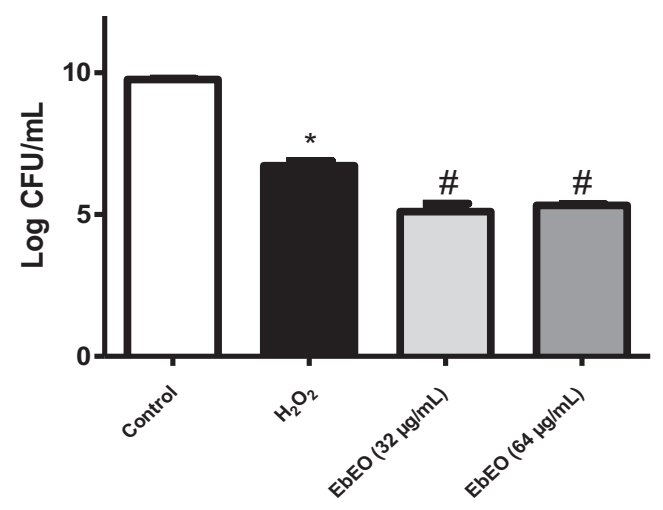

FIGURE 4 | Eugenia brejoensis essential oil (EbEO) reduces the staphyloxanthin production and $S$. aureus resistance to hydrogen peroxide. (A) EbEO inhibits the staphyloxanthin production. (B) The survival of S. aureus after oxidative stress is reduced by EbEO. * Indicates significant differences in relation to control cells $(p<0.05)$. \# Indicates significant differences in relation to $\mathrm{H}_{2} \mathrm{O}_{2}$ cells $(p<0.05)$.

the viability of C. elegans when compared with PBS-treated group (data not shown).

\section{EbEO Protects Galleria mellonella Larvae Against the $S$. aureus Infection}

The antibacterial activity of EbEO was further analyzed using G. mellonella larvae. EbEO treatment did not show any toxicity to these larvae, resulting in survival curves similar to those observed to PBS-treated animals (data not shown). The larvae infected with S. aureus showed $100 \%$ mortality in the third day and this group exhibited a median survival of 1 day. On the other hand, the treatment with a single dose of EbEO $(6.4 \mathrm{mg} / \mathrm{kg})$ protected $70 \%$ of the larvae at the end of the experiment (4 dpi) (Figure 6A).

Interestingly, EbEO treatment induced small effects on the bacterial load when compared to initial bacterial inoculum 
A

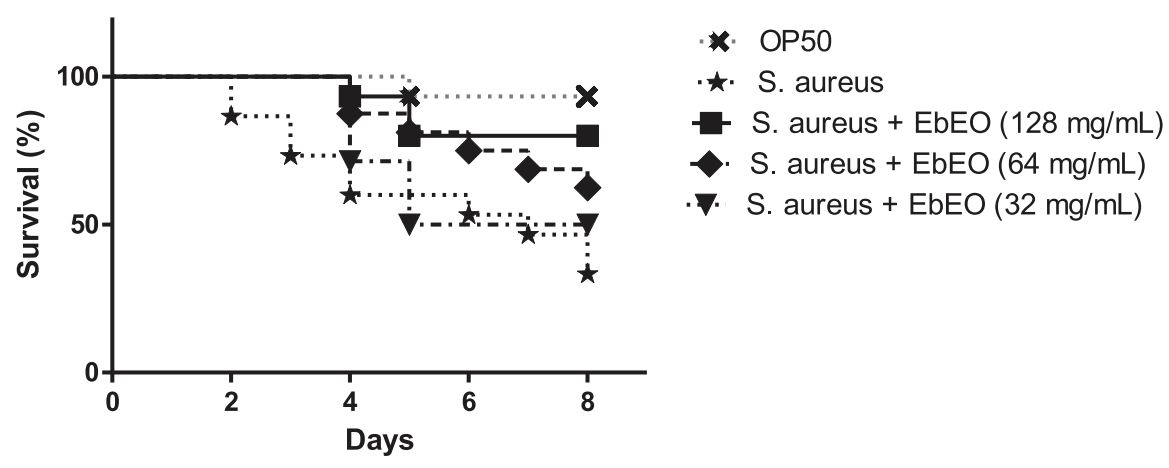

B

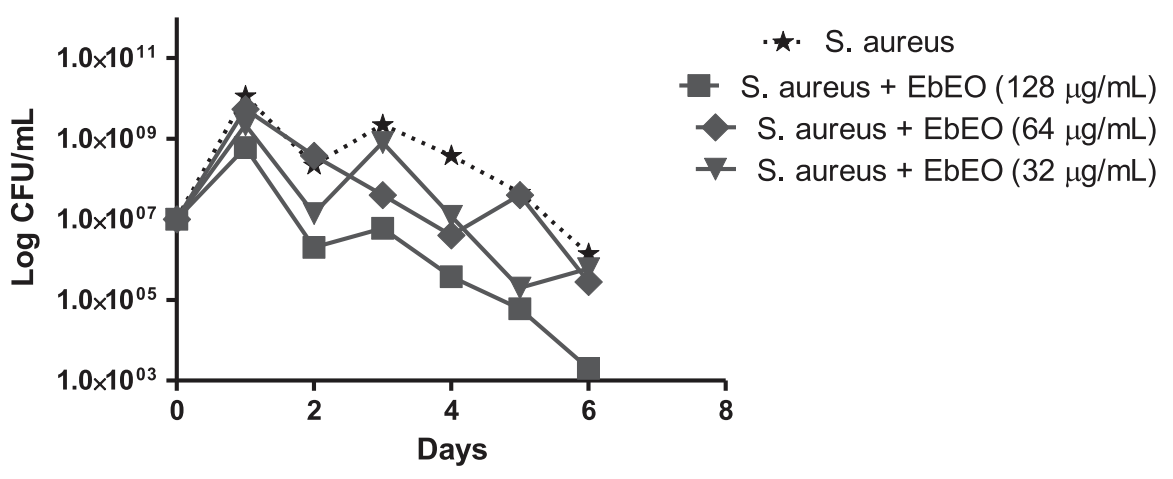

FIGURE 5 | Eugenia brejoensis essential oil (EbEO) protects Caenorhabditis elegans larvae from Staphylococcus aureus infection. (A) Effects of EbEO on Survival of C. elegans larvae infected with S. aureus. (B) Bacterial load. * Indicates significant differences in relation to control cells $(p<0.05)$.

(time 0), suggesting a bacteriostatic action (Figure 6B). In relation to $S$. aureus-infected larvae, EbEO-treated animals exhibited significantly lower levels of bacteria in hemolymph in all days evaluated (around $3 \log \mathrm{CFU} / \mathrm{mL}$ reductions for all days; $p<0.05)$.

Finally, we evaluated whether EbEO could reduce the overproduction of melanin induced by $S$. aureus infection (Figures 6C,D). We observed that the levels of melanin in the hemolymph of $S$. aureus-infected larvae were significantly increased when compared to PBS-treated group (approximately eightfolds after 1 and $3 \mathrm{~h}$ of infection; Figures 6C,D). The treatment with EbEO also significantly reduced the melanin content in hemolymph of G. mellonella larvae at both tested period (reductions of 42.19 and $55.41 \%$ after 1 and $3 \mathrm{~h}$ of infection, respectively; Figures 6C,D).

\section{DISCUSSION}

Due the high chemical diversity exhibited by EOs, they have been pointed out as interesting source of lead molecules for development of alternative antimicrobial therapies (Rubini et al., 2018; Espinoza et al., 2019; Souza Dos Santos et al., 2019). These studies have encouraged the search of new EOs from unexploited plants, and one current example is E. brejoensis a plant recently described in Brazil (Mazine and Souza, 2008; Giaretta and Peixoto, 2014). Herein, we reported the activity of this oil against $S$. aureus, a pathogen highlighted due its ability to harbor genes related with virulence factors and drug resistance pathways (Kos et al., 2012; Berube and Bubeck Wardenburg, 2013; Vestergaard et al., 2019).

EbEO was able to inhibit the $S$. aureus strains with $\mathrm{MIC}_{50}$ of $256 \mu \mathrm{g} / \mathrm{mL}$. As mentioned above, EbEO is mainly composed by sesquiterpenes such as $\delta$-cadinene, $\beta$-caryophyllene, $\alpha$-muurolol, $\alpha$-cadinol, and bicyclogermacrene (Da Silva et al., 2015). The anti-S. aureus activity of EOs containing these sesquiterpenes is well documented in the literature (Mulyaningsih et al., 2010; Salleh et al., 2015; Guerrini et al., 2016; Nishanbaev et al., 2018; Salem et al., 2018). Our results from time-kill studies demonstrated that EbEO is an antibacterial agent showing a synergetic interaction with ampicillin, chloramphenicol, and kanamycin; and additive effects with ciprofloxacin and erythromycin. The improvement of antibiotic action has been reported for several EOs and isolated sesquiterpenes (Langeveld et al., 2014; Lahmar et al., 2017; Espinoza et al., 2019; Khoury et al., 2019).

The overuse and misuse of antibiotics has resulted in different gradient of drug concentrations in humans, animals, and environment (Andersson and Hughes, 2014; Huijbers et al., 2019). In this sense, bacteria are frequently exposed to SubMIC of the antimicrobial agent and these conditions could result in mutagenesis and release of microbial virulence factors (Andersson and Hughes, 2014; Jo and Ahn, 2016; Larsen et al., 2016; Huijbers et al., 2019). Thus, it is important to evaluate 
A
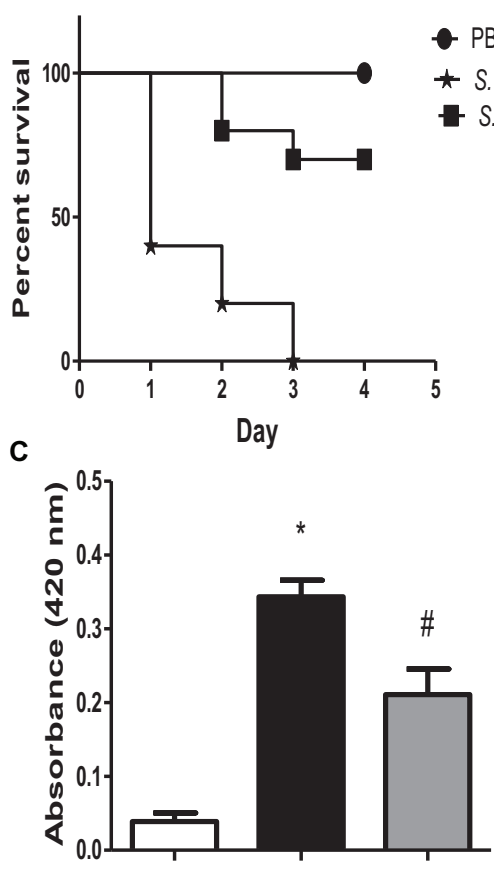

$\beta^{\beta}$

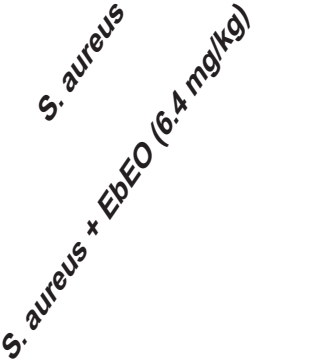

B
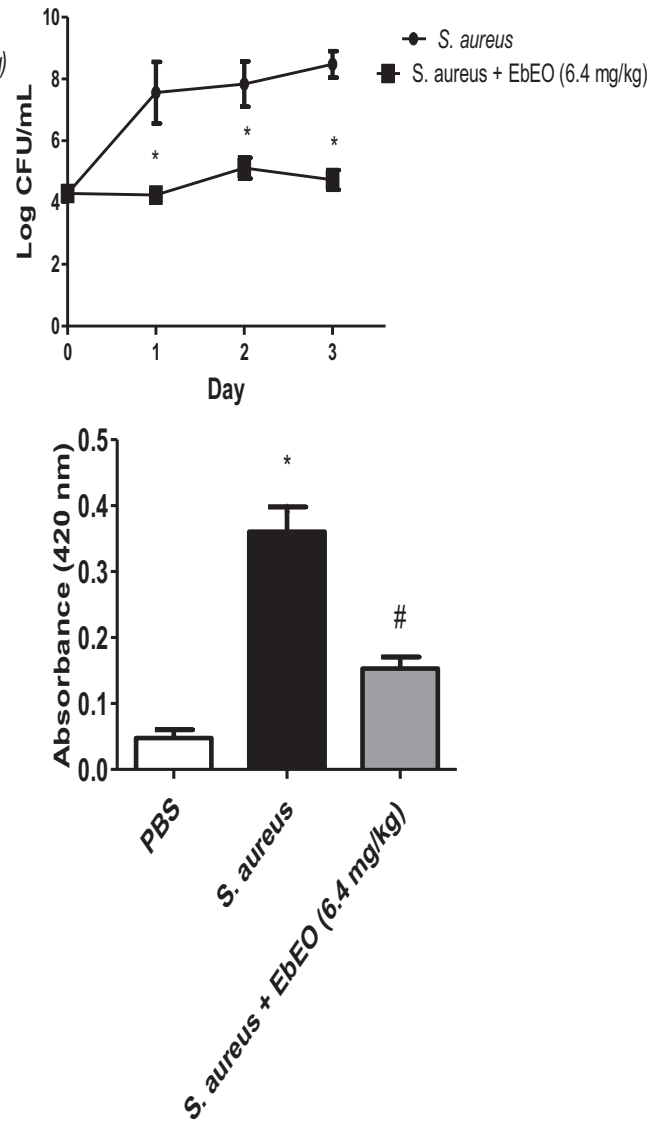

FIGURE 6 | Effects of Eugenia brejoensis essential oil (EbEO) on Galleria mellonella infected with S. aureus. (A) EbEO improves the survival of G. mellonella infected with S. aureus. (B) EbEO reduces the load of $S$. aureus in hemolymph. (C) Effects of EbEO on melanin production after 1 h of $S$. aureus-infection. (D) Effects of EbEO on melanin production after $3 \mathrm{~h}$ of $S$. aureus-infection. * Indicates significant differences in relation to uninfected larvae $(p<0.05)$. \# Indicates significant differences in relation to uninfected and $S$. aureus-infected larvae $(p<0.05)$.

the effects of the antimicrobial agents in pathways related to mutagenesis and virulence (Hobdey et al., 2017; Duan et al., 2018). We first showed that EbEO did not induce the expression of $\operatorname{rec} A$ which is the first gene in the SOS response. The activation of SOS response occurs when bacteria need to repair damages induced in the DNA due antibiotic treatment and other adverse conditions (Simmons et al., 2008; Silva et al., 2017). The activation of SOS pathway is associated with emergence of drug resistance and acquisition of virulence factors (Andreoni et al., 2019; Meunier et al., 2019).

Our data also report that EbEO interferes with the regulation of the accessory gene regulator $(a g r)$-mediated quorum sensing. This system upregulates the production of secreted virulence factors (such as $\alpha$-hemolysin encoded by hla) and downregulates cell surface proteins (such as protein A encoded by spa) (Gottschalk et al., 2013; Singh and Ray, 2014). We demonstrated that sub-inhibitory concentrations of EbEO decreased the expression of hla and increased the expression of spa. Further, $S$. aureus grown in the presence of EbEO displayed reduced hemolytic activity and less ability to survival in whole blood. $\alpha$-Hemolysin induces toxicity toward a broad range of cells and is associated to severe injuries in cutaneous (skin necrosis) and systemic infections (Berube and Bubeck Wardenburg, 2013; Geoghegan et al., 2018; Surewaard et al., 2018).

Other antivirulence effect of EbEO is the inhibition of STX production, a carotenoid from $S$. aureus membrane that confers protection against reactive oxygen species produced by host defense, allowing the bacteria to persist in the inflammation site (Liu and Nizet, 2009; Pannu et al., 2019). These properties make STX an attractive target for antivirulence therapy as demonstrated for some plant-derived compounds (Lee et al., 2013; Silva et al., 2017; Colasso et al., 2019). The inhibitory effects of EbEO toward STX production could be confirmed by the fact that $S$. aureus grown in the presence of this oil showed increased 
susceptibility to hydrogen peroxide. In addition, it may also relate to the efficacy of EbEO to rescue C. elegans and G. mellonella from $S$. aureus infection.

Caenorhabditis elegans is free-living terrestrial nematode considered a fast, cheap, and efficient model for in vivo testing of antimicrobial substances since it is susceptible to human pathogens such as S. aureus (Kong et al., 2014, 2016a). Our results showed that the treatment with EbEO increased the worm lifespam and decreased the bacterial load. The anti-infective efficacy of EbEO was also demonstrated using G. mellonella larvae. EbEO-treated animals also showed lower levels of melanin, a soluble molecule part of the humoral response of $G$. mellonella (together with lysozyme, antimicrobial peptides, and opsonins) (Zdybicka-Barabas et al., 2013; Tsai et al., 2016). The melanogenesis is part of prophenoloxidase cascade which is activated by pathogens and other foreign particles leading accumulation of nodules in order to control the microbial replication (Trevijano-Contador and Zaragoza, 2018). The melanin overproduction results in high levels of cytotoxic compounds that may induce serious damage to host tissues and cells (Zdybicka-Barabas et al., 2014). In this sense, the oil could also protect the larvae against the deleterious effects related to $S$. aureus infection.

Taken together, the results indicated the EO of E. brejoensis is an important source of molecules with anti-S. aureus action, even against MDR strains. Importantly, the oil reduced the expression of significant factors involved in S. aureus virulence and impaired the ability of this bacterium to survive in adverse conditions such as whole blood and under oxidative stress. The antimicrobial efficacy was confirmed using two alternative models of infections based on C. elegans and G. mellonella. These data denote the importance of prospecting new species from underexploited environmental (such as Brazilian caatinga)

\section{REFERENCES}

Alves, J. C. O., Ferreira, G. F., Santos, J. R., Silva, L. C. N., Rodrigues, J. F. S., Neto, W. R. N., et al. (2017). Eugenol induces phenotypic alterations and increases the oxidative burst in Cryptococcus. Front. Microbiol. 8:2419. doi: 10.3389/fmicb. 2017.02419

Andersson, D. I., and Hughes, D. (2014). Microbiological effects of sublethal levels of antibiotics. Nat. Rev. Microbiol. 12, 465-478. doi: 10.1038/nrmicro3270

Andreoni, F., Toyofuku, M., Menzi, C., Kalawong, R., Mairpady Shambat, S., Francois, P., et al. (2019). Antibiotics stimulate formation of vesicles in Staphylococcus aureus in both phage-dependent and -independent fashions and via different routes. Antimicrob. Agents Chemother. 63:e01439-18. doi: 10.1128/ AAC.01439-18

Asgeirsson, H., Thalme, A., and Weiland, O. (2018). Staphylococcus aureus bacteraemia and endocarditis - epidemiology and outcome: a review. Infect Dis. 50, 175-192.

Bakthavatchalam, Y. D., Veeraraghavan, B., Devanga Ragupathi, N. K., Babu, P., Munuswamy, E., and David, T. (2017). Draft genome sequence of reduced teicoplanin-susceptible and vancomycin-heteroresistant methicillin-resistant Staphylococcus aureus from sepsis cases. J. Glob. Antimicrob. Resist. 8, 169-171. doi: 10.1016/j.jgar.2016.12.008

Berube, B. J., and Bubeck Wardenburg, J. (2013). Staphylococcus aureus alphatoxin: nearly a century of intrigue. Toxins 5, 1140-1166. doi: 10.3390/ toxins 5061140

Capone, A., Cafiso, V., Campanile, F., Parisi, G., Mariani, B., Petrosillo, N., et al. (2016). In vivo development of daptomycin resistance in in order to identify new lead molecules for antimicrobial therapy. Further testing is required to know the mode of action of EbEO and details about the action of this oil against the virulence of $S$. aureus as well as individual role of the main components of the EO of E. brejoensis are essential for antimicrobial action.

\section{DATA AVAILABILITY STATEMENT}

The raw data supporting the conclusions of this article will be made available by the authors, without undue reservation, to any qualified researcher.

\section{AUTHOR CONTRIBUTIONS}

$\mathrm{CB}$ and LS contributed to conceptualization and investigation. $\mathrm{AL}-\mathrm{O}, \mathrm{KK}, \mathrm{MC}$, and MV contributed to resources. CB, LS, AL-O, and CS contributed to investigation and formal analysis. $\mathrm{CB}, \mathrm{LS}$, $\mathrm{MV}, \mathrm{MC}, \mathrm{MS}$, and KK contributed to writing original draft. CB, $\mathrm{MV}$, and LS contributed to supervision, writing-review, editing, and project administration.

\section{FUNDING}

This work was supported by the Fundação de Amparo à Pesquisa do Estado de São Paulo (FAPESP) (Proc.2017/066307); Coordenação de Aperfeiçoamento de Pessoal de Nível Superior-Brasil (CAPES)-Finance Code 001; Conselho Nacional de Desenvolvimento Científico e Tecnológico (CNPq) (401452/2016-6).

vancomycin-susceptible methicillin-resistant Staphylococcus aureus severe infections previously treated with glycopeptides. Eur. J. Clin. Microbiol. Infect. Dis. 35, 625-631. doi: 10.1007/s10096-016-2581-4

Colasso, A. H. M., Barros, T. F., Figueiredo, I., Carvalho Junior, A. R., Fernandes, E. S., Uchoa, M. R. B., et al. (2019). The latex of Euphorbia tirucalli inhibits staphyloxanthin production and protects Tenebrio molitor larvae against Staphylococcus aureus infection. Nat. Prod. Res. doi: 10.1080/14786419.2019. 1582036 [Epub ahead of print].

Da Silva, A. G., Alves, R. C. C., Filho, C. M. B., Bezerra-Silva, P. C., Santos, L. M. M. D., Foglio, M. A., et al. (2015). Chemical composition and larvicidal activity of the essential oil from leaves of Eugenia brejoensis Mazine (Myrtaceae). J. Essent. Oil Bear. Plants 18, 1441-1447. doi: 10.1080/0972060x. 2014.1000390

Da Silva, A. P., Nascimento Da Silva, L. C., Martins Da Fonseca, C. S., De Araujo, J. M., Correia, M. T., Cavalcanti Mda, S., et al. (2016). Antimicrobial activity and phytochemical analysis of organic extracts from cleome spinosa Jaqc. Front. Microbiol. 7:963. doi: 10.3389/fmicb.2016.00963

De Carvalho, S. P., De Almeida, J. B., De Freitas, L. M., Guimaraes, A. M. S., Do Nascimento, N. C., Dos Santos, A. P., et al. (2019). Genomic profile of Brazilian methicillin-resistant Staphylococcus aureus resembles clones dispersed worldwide. J. Med. Microbiol. 68, 693-702. doi: 10.1099/jmm.0.00 0956

Dos Santos, B. S., Da Silva, L. C., Da Silva, T. D., Rodrigues, J. F., Grisotto, M. A., Correia, M. T., et al. (2016). Application of omics technologies for evaluation of antibacterial mechanisms of action of plant-derived products. Front. Microbiol. 7:1466. 
Duan, J., Li, M., Hao, Z., Shen, X., Liu, L., Jin, Y., et al. (2018). Subinhibitory concentrations of resveratrol reduce alpha-hemolysin production in Staphylococcus aureus isolates by downregulating saeRS. Emerg. Microbes Infect. 7:136. doi: 10.1038/s41426-018-0142-x

Endimiani, A., Blackford, M., Dasenbrook, E. C., Reed, M. D., Bajaksouszian, S., Hujer, A. M., et al. (2011). Emergence of linezolid-resistant Staphylococcus aureus after prolonged treatment of cystic fibrosis patients in Cleveland, Ohio. Antimicrob. Agents Chemother. 55, 1684-1692. doi: 10.1128/AAC.0130 8-10

Espinoza, J., Urzua, A., Sanhueza, L., Walter, M., Fincheira, P., Munoz, P., et al. (2019). Essential oil, extracts, and sesquiterpenes obtained from the heartwood of pilgerodendron uviferum act as potential inhibitors of the Staphylococcus aureus NorA multidrug efflux pump. Front. Microbiol. 10:337. doi: 10.3389/ fmicb.2019.00337

Geoghegan, J. A., Irvine, A. D., and Foster, T. J. (2018). Staphylococcus aureus and atopic dermatitis: a complex and evolving relationship. Trends Microbiol. 26, 484-497. doi: 10.1016/j.tim.2017.11.008

Giaretta, A., and Peixoto, A. L. (2014). New records of Eugenia brejoensis Mazine (Myrtaceae) and complementary description. Check List 10, 1176-1178. doi: $10.15560 / 10.5 .1176$

Gottschalk, S., Ifrah, D., Lerche, S., Gottlieb, C. T., Cohn, M. T., Hiasa, H., et al. (2013). The antimicrobial lysine-peptoid hybrid LP5 inhibits DNA replication and induces the SOS response in Staphylococcus aureus. BMC Microbiol. 13:192. doi: 10.1186/1471-2180-13-192

Guerrini, A., Sacchetti, G., Grandini, A., Spagnoletti, A., Asanza, M., and Scalvenzi, L. (2016). Cytotoxic effect and TLC bioautography-guided approach to detect health properties of amazonian Hedyosmum sprucei essential oil. Evid. Based Complement. Alternat. Med. 2016:1638342. doi: 10.1155/2016/1638342

Hobdey, S. E., Katahira, E. J., Dockstader, P., Davidson, S. M., Bond, L., Bolz, D. D., et al. (2017). Subinhibitory dalbavancin attenuates exotoxin production from methicillin-sensitive and methicillin-resistant Staphylococcus aureus in vitro. Antimicrob. Agents Chemother. 61:e01090-17. doi: 10.1128/AAC.01090-17

Huijbers, P. M. C., Flach, C. F., and Larsson, D. G. J. (2019). A conceptual framework for the environmental surveillance of antibiotics and antibiotic resistance. Environ. Int. 130:104880. doi: 10.1016/j.envint.2019.05.074

Jakobsen, H., Bojer, M. S., Marinus, M. G., Xu, T., Struve, C., Krogfelt, K. A., et al. (2013). The alkaloid compound harmane increases the lifespan of Caenorhabditis elegans during bacterial infection, by modulating the nematode's innate immune response. PLoS One 8:e60519. doi: 10.1371/journal.pone. 0060519

Jo, A., and Ahn, J. (2016). Phenotypic and genotypic characterisation of multiple antibiotic-resistant Staphylococcus aureus exposed to subinhibitory levels of oxacillin and levofloxacin. BMC Microbiol. 16:170. doi: 10.1186/s12866-016$0791-7$

Kang, J., Liu, L., Wu, X., Sun, Y., and Liu, Z. (2018). Effect of thyme essential oil against Bacillus cereus planktonic growth and biofilm formation. Appl. Microbiol. Biotechnol. 102, 10209-10218. doi: 10.1007/s00253-018-9401-y

Khoury, M., El Beyrouthy, M., Ouaini, N., Eparvier, V., and Stien, D. (2019). Hirtellina lobelii DC. essential oil, its constituents, its combination with antimicrobial drugs and its mode of action. Fitoterapia 133, 130-136. doi: 10.1016/j.fitote.2019.01.001

Kim, Y. G., Lee, J. H., Gwon, G., Kim, S. I., Park, J. G., and Lee, J. (2016). Essential oils and eugenols inhibit biofilm formation and the virulence of Escherichia coli O157: H7. Sci. Rep. 6:36377. doi: 10.1038/srep36377

Kong, C., Eng, S. A., Lim, M. P., and Nathan, S. (2016a). Beyond traditional antimicrobials: a Caenorhabditis elegans model for discovery of novel antiinfectives. Front. Microbiol. 7:1956.

Kong, C., Neoh, H. M., and Nathan, S. (2016b). Targeting Staphylococcus aureus toxins: a potential form of anti-virulence therapy. Toxins 8:E72. doi: 10.3390/ toxins 8030072

Kong, C., Yehye, W. A., Abd Rahman, N., Tan, M. W., and Nathan, S. (2014). Discovery of potential anti-infectives against Staphylococcus aureus using a Caenorhabditis elegans infection model. BMC Complement. Altern. Med. 14:4. doi: 10.1186/1472-6882-14-4

Kos, V. N., Desjardins, C. A., Griggs, A., Cerqueira, G., Van Tonder, A., Holden, M. T., et al. (2012). Comparative genomics of vancomycin-resistant Staphylococcus aureus strains and their positions within the clade most commonly associated with Methicillin-resistant S. aureus hospital-acquired infection in the United States. mBio 3:e112. doi: 10.1128/mBio.00112-12
Lahmar, A., Bedoui, A., Mokdad-Bzeouich, I., Dhaouifi, Z., Kalboussi, Z., Cheraif, I., et al. (2017). Reversal of resistance in bacteria underlies synergistic effect of essential oils with conventional antibiotics. Microb. Pathog. 106, 50-59. doi: 10.1016/j.micpath.2016.10.018

Langeveld, W. T., Veldhuizen, E. J., and Burt, S. A. (2014). Synergy between essential oil components and antibiotics: a review. Crit. Rev. Microbiol. 40, 76-94. doi: 10.3109/1040841X.2013.763219

Larsen, J., Clasen, J., Hansen, J. E., Paulander, W., Petersen, A., Larsen, A. R., et al. (2016). Copresence of tet(K) and tet(M) in livestock-associated methicillinresistant Staphylococcus aureus clonal complex 398 is associated with increased fitness during exposure to sublethal concentrations of tetracycline. Antimicrob. Agents Chemother. 60, 4401-4403. doi: 10.1128/AAC.00426-16

Lee, J. H., Cho, H. S., Kim, Y., Kim, J. A., Banskota, S., Cho, M. H., et al. (2013). Indole and 7-benzyloxyindole attenuate the virulence of Staphylococcus aureus. Appl. Microbiol. Biotechnol 97, 4543-4552. doi: 10.1007/s00253-012-4674-z

Liang, Y., Tu, C., Tan, C., El-Sayed Ahmed, M. A. E., Dai, M., Xia, Y., et al. (2019). Antimicrobial resistance, virulence genes profiling and molecular relatedness of methicillin-resistant Staphylococcus aureus strains isolated from hospitalized patients in Guangdong Province, China. Infect. Drug Resist. 12, 447-459. doi: 10.2147/IDR.S192611

Liu, G. Y., Essex, A., Buchanan, J. T., Datta, V., Hoffman, H. M., Bastian, J. F., et al. (2005). Staphylococcus aureus golden pigment impairs neutrophil killing and promotes virulence through its antioxidant activity. J. Exp. Med. 202, 209-215. doi: $10.1084 /$ jem.20050846

Liu, G. Y., and Nizet, V. (2009). Color me bad: microbial pigments as virulence factors. Trends Microbiol. 17, 406-413. doi: 10.1016/j.tim.2009.06.006

Mazine, F. F., and Souza, V. C. (2008). A new species of Eugenia (Myrtaceae) from north-eastern Brazil. Bot. J. Linn. Soc. 158, 775-777. doi: 10.1111/j.1095-8339. 2008.00921.x

Mcneil, J. C., and Fritz, S. A. (2019). Prevention strategies for recurrent community-associated Staphylococcus aureus skin and soft tissue infections. Curr. Infect. Dis. Rep. 21:12. doi: 10.1007/s11908-019-0670-0

Mendes, J., Martins, H., Otoni, C., Santana, N., Silva, R., Da Silva, A., et al. (2018). Chemical composition and antibacterial activity of Eugenia brejoensis essential oil nanoemulsions against Pseudomonas fluorescens. LWT 93, 659-664. doi: 10.1016/j.lwt.2018.04.015

Meunier, A., Nerich, V., Fagnoni-Legat, C., Richard, M., Mazel, D., Adotevi, O., et al. (2019). Enhanced emergence of antibiotic-resistant pathogenic bacteria after in vitro induction with cancer chemotherapy drugs. J. Antimicrob. Chemother. 74, 1572-1577. doi: 10.1093/jac/dkz070

Mittal, R. P., Rana, A., and Jaitak, V. (2018). Essential oils: an impending substitute of synthetic antimicrobial agents to overcome antimicrobial resistance. Curr. Drug Targets 20, 605-624. doi: 10.2174/1389450119666181031122917

Mulyaningsih, S., Youns, M., El-Readi, M. Z., Ashour, M. L., Nibret, E., Sporer, F., et al. (2010). Biological activity of the essential oil of Kadsura longipedunculata (Schisandraceae) and its major components. J. Pharm. Pharmacol. 62, 10371044. doi: 10.1111/j.2042-7158.2010.01119.x

Nielsen, A., Nielsen, K. F., Frees, D., Larsen, T. O., and Ingmer, H. (2010). Method for screening compounds that influence virulence gene expression in Staphylococcus aureus. Antimicrob. Agents Chemother. 54, 509-512. doi: 10. 1128/AAC.00940-09

Nishanbaev, S., Bobakulov, K., Okhundedaev, B., Sasmakov, S., Yusupova, E., Azimova, S., et al. (2018). Component composition of the extracts and essential oils from the Alhagi canescens, growing in Uzbekistan and their antimicrobial activity. Nat. Prod. Res. 33, 3417-3420. doi: 10.1080/14786419.2018.1475384

Oliveira De Souza, L. I., Bezzera-Silva, P. C., Do Amaral, Ferraz Navarro, D. M., Da Silva, A. G., Dos Santos, et al. (2017). The chemical composition and trypanocidal activity of volatile oils from Brazilian caatinga plants. Biomed. Pharmacother. 96, 1055-1064. doi: 10.1016/j.biopha.2017.11.121

Pannu, M. K., Hudman, D. A., Sargentini, N. J., and Singh, V. K. (2019). Role of SigB and staphyloxanthin in radiation survival of Staphylococcus aureus. Curr. Microbiol. 76, 70-77. doi: 10.1007/s00284-018-1586-x

Rao, J., Chen, B., and Mcclements, D. J. (2019). Improving the efficacy of essential oils as antimicrobials in foods: mechanisms of action. Annu. Rev. Food Sci. Technol. 10, 365-387. doi: 10.1146/annurev-food-032818-121727

Rubini, D., Banu, S. F., Nisha, P., Murugan, R., Thamotharan, S., Percino, M. J., et al. (2018). Essential oils from unexplored aromatic plants quench biofilm formation and virulence of Methicillin resistant Staphylococcus aureus. Microb. Pathog. 122, 162-173. doi: 10.1016/j.micpath.2018.06.028 
Salem, M. Z. M., El-Hefny, M., Ali, H. M., Elansary, H. O., Nasser, R. A., El-Settawy, A. A. A., et al. (2018). Antibacterial activity of extracted bioactive molecules of Schinus terebinthifolius ripened fruits against some pathogenic bacteria. Microb. Pathog. 120, 119-127. doi: 10.1016/j.micpath.2018. 04.040

Salleh, W. M., Ahmad, F., and Yen, K. H. (2015). Chemical compositions and biological activities of the essential oils of Beilschmiedia madang Blume (Lauraceae). Arch. Pharm. Res. 38, 485-493. doi: 10.1007/s12272-0140460-z

Silva, L. N., Da Hora, G. C. A., Soares, T. A., Bojer, M. S., Ingmer, H., Macedo, A. J., et al. (2017). Myricetin protects Galleria mellonella against Staphylococcus aureus infection and inhibits multiple virulence factors. Sci. Rep. 7:2823. doi: 10.1038/s41598-017-02712-1

Simmons, L. A., Foti, J. J., Cohen, S. E., and Walker, G. C. (2008). The SOS regulatory network. EcoSal Plus 2008, 1-48. doi: 10.1128/ecosalplus.5.4.3

Singh, R., and Ray, P. (2014). Quorum sensing-mediated regulation of staphylococcal virulence and antibiotic resistance. Future Microbiol. 9, 669-681. doi: $10.2217 / \mathrm{fmb} .14 .31$

Souza Dos Santos, B., Bezerra Filho, C. M., Alves Do Nascimento, J. A. Jr., Brust, F. R., Bezerra-Silva, P. C., Lino Da Rocha, S. K., et al. (2019). Anti-staphylococcal activity of Syagrus coronata essential oil: Biofilm eradication and in vivo action on Galleria mellonela infection model. Microb. Pathog. 131, 150-157. doi: 10.1016/j.micpath.2019. 04.009

Stevanovic, Z. D., Bosnjak-Neumuller, J., Pajic-Lijakovic, I., Raj, J., and Vasiljevic, M. (2018). Essential oils as feed additives-future perspectives. Molecules 23:1717. doi: 10.3390/molecules 23071717

Stiernagle, T. (2006). "Maintenance of C. elegans," in WormBook, ed. The C. elegans Research Community (Pasadena, CA: WormBook).

Surewaard, B. G. J., Thanabalasuriar, A., Zeng, Z., Tkaczyk, C., Cohen, T. S., Bardoel, B. W., et al. (2018). Alpha-Toxin induces platelet aggregation and liver injury during Staphylococcus aureus Sepsis. Cell Host Microbe 24:e273. doi: $10.1016 /$ j.chom.2018.06.017

Taghavi, T., Kim, C., and Rahemi, A. (2018). Role of natural volatiles and essential oils in extending shelf life and controlling postharvest microorganisms of small fruits. Microorganisms 6:104. doi: 10.3390/microorganisms60 40104

Trevijano-Contador, N., and Zaragoza, O. (2018). Immune response of Galleria mellonella against human fungal pathogens. J. Fungi 5:E3.
Tsai, C. J., Loh, J. M., and Proft, T. (2016). Galleria mellonella infection models for the study of bacterial diseases and for antimicrobial drug testing. Virulence 7 , 214-229. doi: 10.1080/21505594.2015.1135289

Turner, N. A., Sharma-Kuinkel, B. K., Maskarinec, S. A., Eichenberger, E. M., Shah, P. P., Carugati, M., et al. (2019). Methicillin-resistant Staphylococcus aureus: an overview of basic and clinical research. Nat. Rev. Microbiol. 17, 203-218. doi: 10.1038/s41579-018-0147-4

Vestergaard, M., Frees, D., and Ingmer, H. (2019). Antibiotic resistance and the MRSA problem. Microbiol. Spectr. 7:GPP3-0057-2018. doi: 10.1128/ microbiolspec.GPP3-0057-2018

Vestergaard, M., Paulander, W., and Ingmer, H. (2015). Activation of the SOS response increases the frequency of small colony variants. BMC Res. Notes 8:749. doi: 10.1186/s13104-015-1735-2

Werth, B. J., Barber, K. E., Ireland, C. E., and Rybak, M. J. (2014). Evaluation of ceftaroline, vancomycin, daptomycin, or ceftaroline plus daptomycin against daptomycin-nonsusceptible methicillin-resistant Staphylococcus aureus in an in vitro pharmacokinetic/pharmacodynamic model of simulated endocardial vegetations. Antimicrob. Agents Chemother. 58, 3177-3181. doi: 10.1128/AAC. 00088-14

Zdybicka-Barabas, A., Mak, P., Jakubowicz, T., and Cytrynska, M. (2014). Lysozyme and defense peptides as suppressors of phenoloxidase activity in Galleria mellonella. Arch. Insect Biochem. Physiol. 87, 1-12. doi: 10.1002/arch. 21175

Zdybicka-Barabas, A., Staczek, S., Mak, P., Skrzypiec, K., Mendyk, E., and Cytrynska, M. (2013). Synergistic action of Galleria mellonella apolipophorin III and lysozyme against Gram-negative bacteria. Biochim. Biophys. Acta 1828, 1449-1456. doi: 10.1016/j.bbamem.2013.02.004

Conflict of Interest: The authors declare that the research was conducted in the absence of any commercial or financial relationships that could be construed as a potential conflict of interest.

Copyright $\odot 2020$ Bezerra Filho, da Silva, da Silva, Løbner-Olesen, Struve, Krogfelt, Correia and Vilela Oliva. This is an open-access article distributed under the terms of the Creative Commons Attribution License (CC BY). The use, distribution or reproduction in other forums is permitted, provided the original author(s) and the copyright owner(s) are credited and that the original publication in this journal is cited, in accordance with accepted academic practice. No use, distribution or reproduction is permitted which does not comply with these terms. 